\title{
Catalyst Selection Facilitates the use of Heterocyclic Sulfinates as General Nucleophilic Coupling Partners in Palladium-Catalyzed Coupling Reactions
}

\author{
Tim Markovic, ${ }^{a}$ Benjamin N. Rocke, ${ }^{b}$ David C. Blakemore, ${ }^{b}$ Vincent Mascitti ${ }^{b}$ and Michael C. Willis ${ }^{a, *}$ \\ ${ }^{a}$ Department of Chemistry, University of Oxford, Chemistry Research Laboratory, Mansfield Road, Oxford, OX1 3TA, U.K. \\ ${ }^{b}$ Medicine Design, Pfizer Inc., Eastern Point Road, Groton, CT 06340, USA.
}

Supporting Information Placeholder

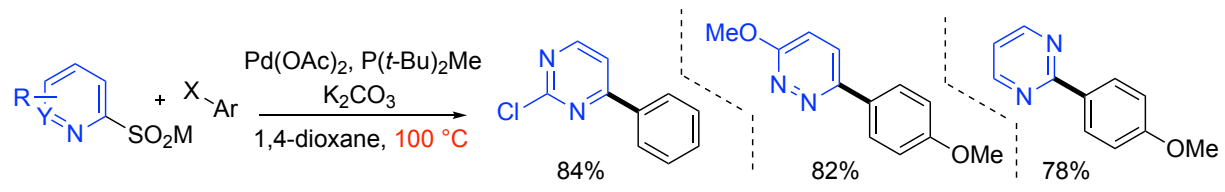

\begin{abstract}
A range of 5- and 6-membered heterocycle-derived sulfinates are shown to be effective nucleophilic coupling partners with aryl chlorides and bromides using $\operatorname{Pd}(0)$ catalysis. The use of optimal reaction conditions, specifically incorporating a $\mathrm{P}(t$ $\mathrm{Bu})_{2} \mathrm{Me}$-derived Pd catalyst, allowed reactions to be performed at moderate temperatures and enabled the inclusion of a variety of sensitive functional groups. Challenging heterocyclic sulfinates, including pyrazine, pyridazine, pyrimidine, pyrazole and imidazole were all shown to perform well.
\end{abstract}

The ability to use cross-coupling methods to access substituted heteroaromatics is crucial to medicinal chemistry, where functionalized heterocycles are key components of many target structures. ${ }^{1}$ However, heteroaromatic reagents are notoriously challenging substrates for a variety of cross-coupling reactions. One of the most documented challenges is the poor performance of heterocycle-derived boronic acids, and related boronates, in palladium-catalyzed Suzuki-Miyaura reactions. ${ }^{2}$ Several factors contribute to these difficulties, but the propensity of heteroaryl boronic acid derivatives to undergo rapid proto-deboronation ${ }^{3}$ is the major problem. ${ }^{4} \mathrm{~N}$-Heterocycles are notorious in this regard. To address these issues we recently reported on the preparation and use of pyridine sulfinates as replacements for pyridine boronates in palladium-catalyzed coupling reactions with aryl halides. ${ }^{5}$ Pyridine sulfinates are straightforward to prepare and stable under extended storage times, and conductive to high-yielding coupling reactions when combined with a broad range of aryl halides (Scheme 1). Despite the success of this chemistry there were some notable constraints, due to the elevated reaction temperature required $\left(150{ }^{\circ} \mathrm{C}\right)$ and the corresponding functional group compatibility limitations. ${ }^{6}$ The use of elevated temperatures has also been noted by others as being a potential drawback from a process chemistry perspective. ${ }^{7}$ In this Letter we show how the use of an alternative catalyst allows the reaction temperature to be dramatically reduced, thus delivering improved functional group tolerance. We also report the preparation and successful coupling chemistry of a broad range of 5- and 6-ring heteroaromatic sulfinates.
Scheme 1. Heterocylic boronic acids versus heterocyclic sulfinates in coupling reactions and an example pyridine-2sulfinate coupling reaction.

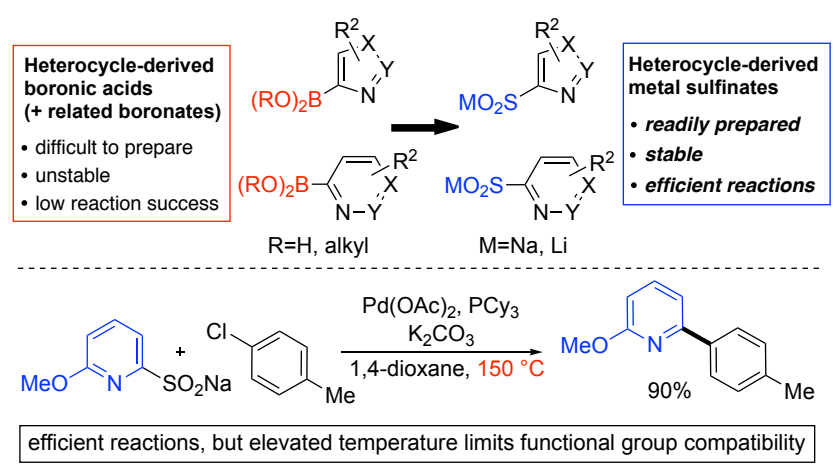

In order to address the stated limitations, we undertook a systematic evaluation of all reaction parameters (Scheme 2). We hypothesized that a more active catalyst would permit lower reaction temperatures and, with our previous results in hand, selected a set of seven ligands including and close-in to the existing high-performers as well as seven ligands that represented unexplored space for further screening. We also selected for evaluation (1) a range of solvents with boiling points above the screening temperature; (2) bases and $\mathrm{Pd}$ sources having shown promise in our previous screening data; and (3) a phase transfer catalyst (PTC) to understand its possible influence on this heterogeneous reaction. Screening was conducted against both chloro and bromo electrophiles in plate format at $100{ }^{\circ} \mathrm{C}$, a temperature that we considered to be reasonable for use in both array and large-scale reactions. Analysis of the screening results identified $\mathrm{P}(t-\mathrm{Bu})_{2} \mathrm{Me}$ (used as its $\mathrm{HBF}_{4}$ salt) as a highly active ligand, affording analytical yields 
up to $87 \%{ }^{8}$ Despite the thorough screening, we resettled on $\mathrm{Pd}(\mathrm{OAc})_{2}, \mathrm{~K}_{2} \mathrm{CO}_{3}$ and dioxane. Validation of these results in singleton experiments performed on preparative scale $(0.2$ mmol) delivered isolated yields of $90 \%$ for coupling with the aryl bromide, and $85 \%$ for the chloride, respectively. A 1.0 mmol scale reaction using the Br-quinoline coupling partner delivered the coupled product in $87 \%$ yeield.

\section{Scheme 2. Catalyst and reaction optimization.}

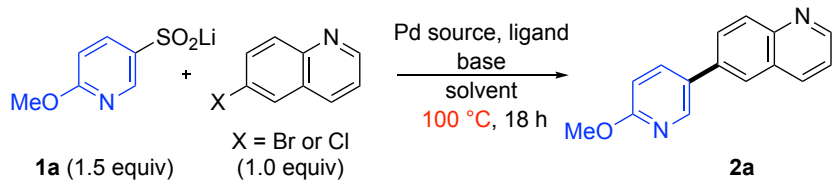

\begin{tabular}{|c|c|c|c|c|c|}
\hline \multicolumn{4}{|c|}{ optimization: 192 experiments $\longrightarrow$} & \multicolumn{2}{|c|}{$87 \%$ analytical yield } \\
\hline \multicolumn{3}{|l|}{ ligands } & \multirow{3}{*}{$\begin{array}{l}\text { solvents } \\
\text { 1,4-dioxane } \\
t \text {-AmOH }\end{array}$} & \multirow{3}{*}{\begin{tabular}{|l|} 
base \\
$\mathrm{K}_{2} \mathrm{CO}_{3}$ \\
$\mathrm{Cs}_{2} \mathrm{CO}_{3}$ \\
\end{tabular}} & \multirow{3}{*}{\begin{tabular}{|l} 
additive \\
TBAB \\
\end{tabular}} \\
\hline $\mathrm{PCy}_{3}$ & $\mathrm{P}(i-\mathrm{Bu})_{3}$ & $-\mathrm{Bu})_{2} \mathrm{Cy}$ & & & \\
\hline $\mathrm{P}(\mathrm{Me})_{3}$ & $\mathrm{P}(t-\mathrm{Bu})_{2} \mathrm{Me}$ & dcpf & & & \\
\hline dtbpf & dippf & QPhos & $\mathrm{EtCN}$ & \multirow{2}{*}{\multicolumn{2}{|c|}{\begin{tabular}{|l|} 
Pd source \\
$\mathrm{Pd}(\mathrm{OAc})_{2}$ \\
$\mathrm{Pd}(\mathrm{MeCN})_{2} \mathrm{OTs}_{2}$ \\
\end{tabular}}} \\
\hline dtbpp & AmPhos & dpFpe & DMF & & \\
\hline \multicolumn{3}{|c|}{$t$-BuBrettPhos phosphoramidite } & & & \\
\hline
\end{tabular}

To test the generality of these newly optimized reaction conditions and ligand selection, we evaluated the coupling of ten pyridine 2-sulfinates, featuring a variety of substitution patterns, with simple aryl halides (Scheme 3). High yielding reactions were achieved in all cases, with both aryl chloride and aryl bromide coupling partners performing well under these new conditions. Importantly, the yields obtained in these coupling reactions were comparable to those achieved with our earlier, higher temperature, conditions. ${ }^{5}$

Having validated these new reaction conditions we then wanted to test our hypothesis that a more active catalyst and milder reaction conditions would allow for greater functional group tolerance. Accordingly, a trifluoromethyl-substituted pyridine-2-sulfinate, and a methoxy-substituted pyridine-3sulfinate were combined with a selection of functionalized coupling partners, all of which had failed under our original reaction conditions (Scheme 4). Pleasingly, a broad range of reactive functional groups were shown to be compatible with the reaction conditions, delivering coupled products in good to excellent yields. Specifically, acidic $\mathrm{OH}$ and NH groups, present in phenol (2l), carboxylic acid (2m), carbamate (2n), sulfonamide (2o) and amide (2p) substituted arenes, were all tolerated. Sulfone (2q) and cyano-containing substrates (2r, 2s) also performed well. A 4-bromo- $N$-methyl pyrazole coupling partner could also be employed (2t), although in this case a reaction temperature of $130{ }^{\circ} \mathrm{C}$ was needed. 2,2'Bipyridine $\mathbf{2 u}$ was obtained in $73 \%$ yield, establishing the validity of these newconditions to promote challenging heterocycle-heterocycle coupling reactions. Medicinally relevant pentafluorosulfide groups were also tolerated (2v). Finally, a sterically demanding 2,6-dimethyl-substituted benzene underwent efficient coupling (2w).
Scheme 3. Evaluation of pyridine-2-sulfinate coupling partners. ${ }^{a}$

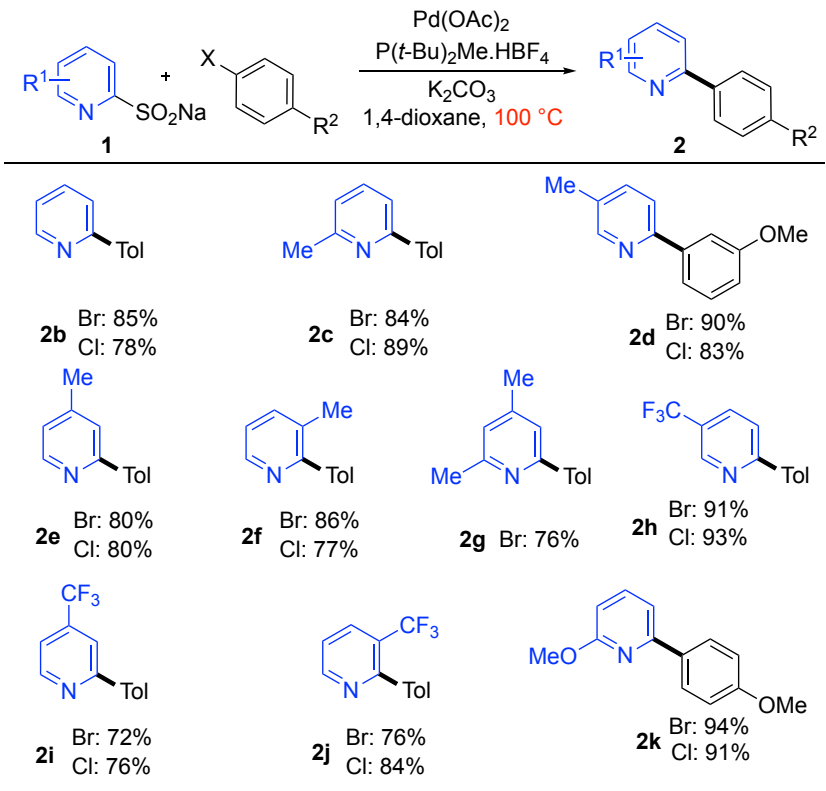

${ }^{a}$ Reaction conditions: Pyridine sulfinate (1.5 equiv), aryl halide (1.0 equiv), $\mathrm{Pd}(\mathrm{OAc})_{2}(5 \mathrm{~mol} \%), \mathrm{P}(t-\mathrm{Bu})_{2} \mathrm{Me}(10 \mathrm{~mol} \%), \mathrm{K}_{2} \mathrm{CO}_{3}$ ( 1.5 equiv), 1,4 -dioxane, $100^{\circ} \mathrm{C}, 18$ hours. Isolated yields.

Scheme 4. Demonstration of functional group compatibility. $^{a}$

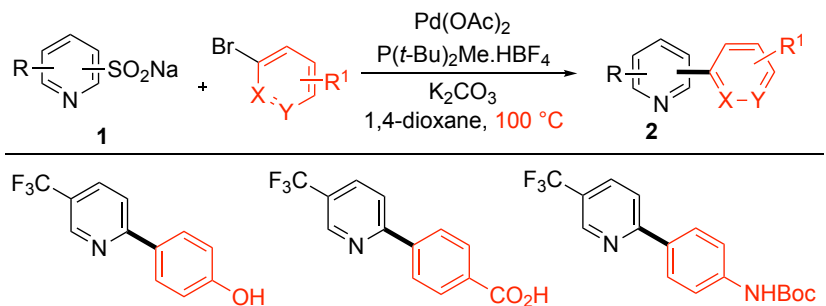

2I, $84 \%$

$2 \mathbf{m},{ }^{b} 87 \%$

2n, $73 \%$

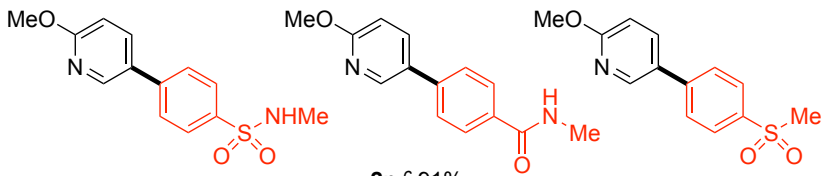

20, ${ }^{c} \mathbf{9 3 \%} \quad 2 \mathbf{2 p},{ }^{c} \mathbf{9 1 \%} \quad \mathbf{2 q},{ }^{c} \mathbf{9 5 \%}$<smiles>N#Cc1ccc(-c2ccc(C(F)(F)F)cn2)cc1</smiles><smiles>COc1ccc(-c2ccc(CN)cn2)cn1</smiles><smiles>COc1ccc(-c2cnn(C)c2)cn1</smiles>

2 r, $90 \%$<smiles>COc1cccc(-c2ccc(C(F)(F)F)cn2)n1</smiles><smiles>COc1ccc(-c2ccc(S(F)(F)F)cc2)cn1</smiles>

$2 \mathbf{t}, c 95 \%$ $\mathrm{F}_{3} \mathrm{C}$<smiles>Cc1cccc(C)c1-c1ccc(I)cn1</smiles>

$2 \mathbf{w}, 94 \%$

${ }^{a}$ Reaction conditions: Pyridine sulfinate (1.5 equiv), aryl halide (1.0 equiv), $\mathrm{Pd}(\mathrm{OAc})_{2}(5 \mathrm{~mol} \%), \mathrm{P}(t-\mathrm{Bu})_{2} \mathrm{Me}(10 \mathrm{~mol} \%), \mathrm{K}_{2} \mathrm{CO}_{3}$ (1.5 equiv), 1,4 -dioxane, $100{ }^{\circ} \mathrm{C}, 18$ hours. Isolated yields. ${ }^{b} \mathrm{I}-$ solated as the methyl ester due to isolation difficulties with the free acid. ${ }^{c}$ Lithium sulfinate used.

Although the challenges of using pyridine-derived boronic acids and related boronates in Suzuki-Miyaura coupling reac- 
tion are well documented, a number of other heterocyclic partners are also known to be particularly problematic. ${ }^{2}$ Consequently, we evaluated the preparation and coupling chemistry of a variety of 5- and 6-membered heterocyclic sulfinates (Scheme 5). ${ }^{9}$ The sulfinates were prepared from either oxidation of the corresponding thiol, ${ }^{10}$ or using SMOPS chemistry from the corresponding halide. ${ }^{11}$ All of the sulfinates used in Scheme 5 are stable solid reagents. Diazene derivatives performed well in the coupling reactions, with pyrazine (3a), pyridazine (3b) and pyrimidine sulfinates $(\mathbf{3 c})$ providing the aryl coupled products in high yields. A 2-quinoline sulfinate produced $3 f$ in $88 \%$ yield. Sulfinates derived from 5membered heterocycles were also effective coupling partners, with 3-indazole (3g), 4-pyrazole (3h), 2-imidazole (3i), and 5indole (3j) substituted sulfinates all delivering efficient reactions.

Scheme 5. 5- and 6-membered heterocyclic sulfinates as nucleophilic coupling partners with aryl bromides. ${ }^{a}$

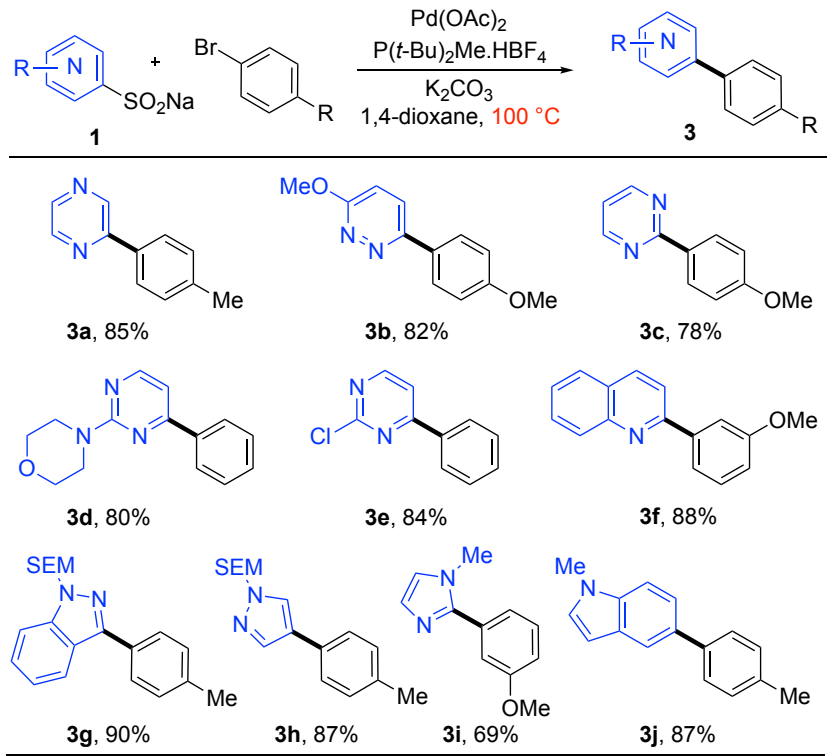

${ }^{a}$ Reaction conditions: Heterocycle sulfinate (1.5 equiv), aryl halide (1.0 equiv), $\mathrm{Pd}(\mathrm{OAc})_{2}(5 \mathrm{~mol} \%), \mathrm{P}(t-\mathrm{Bu})_{2} \mathrm{Me}(10 \mathrm{~mol} \%)$, $\mathrm{K}_{2} \mathrm{CO}_{3}$ (1.5 equiv), 1,4-dioxane, $100{ }^{\circ} \mathrm{C}, 18$ hours. Isolated yields.

In conclusion, we show that the use of a new catalyst system allows the use of lower temperature reaction conditions for the palladium-catalyzed coupling of heteroaryl sulfinates with aryl halides. Excellent functional group tolerance is demonstrated. We also report the preparation and use of a range of medicinally relevant 5 - and 6-membered heterocyclic sulfinates. Given the challenges associated with the use of the corresponding boronic acids, we anticipate that these reagents will find wide use in discovery chemistry.

\section{Supporting Information}

Experimental procedures and full characterization for all compounds. This material is available free of charge via the Internet at http://pubs.acs.org.

\section{AUTHOR INFORMATION}

\section{Corresponding Author}

*michael.willis@chem.ox.ac.uk

\section{ACKNOWLEDGMENT}

We thank the Pfizer and the EPSRC for support of this study. In addition, we acknowledge Greg Steeno, Usa Reilly, and Neal Sach, all of Pfizer Medicine Design, for assistance with reaction screening. BNR, DCB, and VM are employees of Pfizer Inc. and may own stocks in the company.

\section{REFERENCES}

(1) a) Brown, D. G.; Bostrom, J. J. Med. Chem. 2016, 59, 4443; b) Carey, J. S.; Laffan, D.; Thomson, C.; Williams, M. T. Org. Biomol. Chem. 2006, 4, 2337.

(2) Blakemore, D. In Synthetic Methods in Drug Discovery: Volume 1; The Royal Society of Chemistry: 2016; Vol. 1, p 1.

(3) a) Cox, P. A.; Leach, A. G.; Campbell, A. D.; Lloyd-Jones, G. C. J. Am. Chem. Soc. 2016, 138, 9145-9157; b) Cox, P. A.; Reid, M.; Leach, A. G.; Campbell, A. D.; King, E. J.; Lloyd-Jones, G. C. J. Am. Chem. Soc. 2017, 139, 13156-1316.

(4) For alternative solutions to the use of pyridine-2-boronic acids, see: a) Billingsley, K. L.; Buchwald, S. L. Angew. Chem. Int. Ed. 2008, 47, 4695; b) Chen, W.; Zhou, X.; Xiao, F.; Luo, J.; Deng, G. J. Tetrahedron Lett. 2012, 53, 4347; c) Deng, J. Z.; Paone, D. V.; Ginnetti, A. T.; Kurihara, H.; Dreher, S. D.; Weissman, S. A.; Stauffer, S. R.; Burgey, C. S. Org. Lett. 2009, 11, 345; d) Dick, G. R.; Woerly, E. M.; Burke, M. D. Angew. Chem. Int. Ed. 2012, 51, 2667; e) Hodgson, P. B.; Salingue, F. H. Tetrahedron Lett. 2004, 45, 685; f) Ren, W.; Li, J.; Zou, D.; Wu, Y.; Wu, Y. Tetrahedron 2012, 68, 1351; g) Yamamoto, Y.; Takizawa, M.; Yu, X. Q.; Miyaura, N. Angew. Chem. Int. Ed. 2008, 47, 928.

(5) Markovic, T.; Rocke, B. N.; Blakemore, D. C.; Mascitti, V.; Willis, M. C. Chem. Sci. 2017, 8, 4437.

(6) For a review of desulfination in C-C cross-coupling, see: Ortgies, D. H.; Hassanpour, A.; Chen, F.; Woo, S.; Forgione, P. Eur. J. Org. Chem. 2016, 2016, 408.

(7) Ely, R.; Richardson, P.; Zlota, A.; Steven, A.; Kargbo, R.; Nawrat, C. C.; Ramirez, A.; Day, D. P.; Knight, J. Org. Proc. Res. Dev. 2017, 21, 897.

(8) See the Supporting Information for full details.

(9) For thiophene, benzothiophene, furan and benzofuran sulfinates in cross-coupling reactions, see: a) Mangel, D.; Buonomano, C.; Sevigny, S.; Di Censo, G.; Thevendran, G.; Forgione, P. Heterocycles 2015, 90, 1228; b) Sévigny, S.; Forgione, P. Chem. Eur. J. 2013, 19, 2256; c) Sévigny, S.; Forgione, P. New J. Chem. 2013, 37, 589.

(10) Kamiyama, T.; Enomoto, S.; Inoue, M. Chem. Pharm. Bull. 1988, 36, 2652.

(11) Baskin, J. M.; Wang, Z. Tetrahedron Lett. 2002, 43, 8479. 